\title{
Portación nasal de Staphylococcus aureus y sus perfiles de resistencia a antibióticos en niños que viven en zonas de gran altitud del sudoeste de China
}

\author{
Staphylococcus aureus nasal carriage and its antibiotic resistance profiles in \\ children in high altitude areas of Southwestern China
}

\author{
Dra. Zongrong Gong ${ }^{a}$, Dra. Min Shu ${ }^{a}$,Dr. Qing Xia ${ }^{b}$, Dra. Shan Tan ${ }^{a}$ Dr. Wei Zhou ${ }^{c}$, Dra. Yu Zhu $y$ \\ Dra. Chaomin Wan ${ }^{a}$
}

\begin{abstract}
RESUMEN
Antecedentes/Objetivo. Describir el perfil epidemiológico de la portación nasal de cepas de Staphylococcus aureus (S. aureus), su resistencia a antibióticos y la presencia de los genes de leucocidina de Panton-Valentine (LPV) y mecA en niños en edad escolar que viven en zonas de gran altitud del sudoeste de China.

Métodos. En el estudio transversal, se analizaron hisopados nasales de estudiantes a fin de detectar S. aureus. Se realizó la prueba de la reacción en cadena de la polimerasa $(\mathrm{RCP})$ para identificar los genes de LPV y mecA.

Resultados. Del total de 314 niños, se detectó $S$. aureus en el $5,10 \%$ (16/314). La resistencia de las cepas aisladas a la penicilina, eritromicina, clindamicina, rifampicina y cefoxitina fue del $100 \%, 81,3 \%, 81,3 \%, 0,0 \%$ y $6,3 \%$, respectivamente. Ninguna de las cepas mostró resistencia a la vancomicina. Se detectó la expresión del gen mec $A$ en 3 cepas aisladas, y 10 cepas aisladas dieron resultado positivo para el gen de LPV.

Conclusión. Se detectó S. Aureus en el 5,10\% (16/314) de la población delestudio;el0,96\% (3/314) presentóS. Aureus resistente a la meticilina (SARM). Además, se detectó la expresión de los genes de LPV y mecA en 10 y 3 cepas aisladas, respectivamente. Palabras clave: resistencia a antibióticos, niño, Staphylococcus aureus.
\end{abstract}

http:/ / dx.doi.org/10.5546/ aap.2017.274

Texto completo en inglés:

http: / / dx.doi.org/10.5546/ aap.2017.eng.274

Cómo citar: Gong Z, Shu M, Xia Q, et al. Portación nasal de Staphylococcus aureus y sus perfiles de resistencia a antibióticos en niños que viven en zonas de gran altitud del sudoeste de China. Arch Argent Pediatr 2017;115(3):274-277.

a. Departamento de Pediatría, Segundo Hospital Universitario del Oeste de China, Universidad de Sichuan, Chengdú 610041, RepúblicaPopularChina.LaboratorioFundamentaldeDefectos Congénitos y Enfermedades Maternoinfantiles Relacionadas, Ministerio de Educación (Universidad de Sichuan).

b. Departamento de Pediatría, Hospital Popular de Aba, Maerkang 624000, República Popular China.

c. Departamento de Laboratorio Clínico, Segundo Hospital Universitario del Oeste de China, Universidad de Sichuan, Chengdú 610041, República Popular China.

Correspondencia: Dra. Chaomin Wan:wcm0220@126.com

Financiamiento: Este estudio recibió fondos de la Fundación Nacional China de Ciencias Naturales (beca n. ${ }^{\circ} 81102195$ H2610) y de un proyecto a cargo del Departamento de Ciencia y Tecnología de la provincia de Sichuan (beca n 2012JY008).

Conflicto de intereses: Ninguno que declarar.

Recibido: 15-7-2016

Aceptado: 5-12-2016

\section{INTRODUCCIÓN}

El Staphylococcus aureus (S. aureus) es un microorganismo patógeno importante que afecta a niños en todo el mundo, y la prevalencia de Staphylococcus aureus resistente a la meticilina (SARM) es cada vez mayor. ${ }^{1}$ Según un informe, aproximadamente el $80 \%$ de las infecciones por $S$. aureus se debieron a la portación nasal de este microorganismo. ${ }^{2}$ La resistencia a los antibióticos betalactámicos es conferida por una proteína de unión a la penicilina, codificada por el gen $m e c A$, que se encuentra en un elemento cromosómico grande.

Recientemente, se identificó un número creciente de casos de SARM como la causa principal de infecciones extrahospitalarias, por lo general con resultados positivos para los genes de leucocidina de Panton-Valentine (LPV). En condiciones de gran altitud, se cree que ciertos factores climáticos de estos entornos extremos, como la alta radiación UV, la sequía y el frío, hacen que las bacterias desarrollen mecanismos y estrategias eficientes para su proliferación y supervivencia. ${ }^{3}$ Además, la propagación de las bacterias resistentes depende de la comunidad de personas y, en cierta medida, del uso de antibióticos.

China tiene unos 250 millones de kilómetros cuadrados de superficie a gran altitud y una población en esas zonas de aproximadamente 130 millones, por lo que es fundamental conocer la prevalencia de $S$. aureus y su resistencia a antibióticos en esas áreas. La densidad de población y el acceso a la atención médica en las regiones tibetanas son menores que en la China continental. En cierto modo, esto es malo para la proliferación y la propagación de S. aureus. En China, la mayoría de los estudios ${ }^{4,5}$ se llevaron a cabo en niños de la región continental, y solamente uno de esos estudios ${ }^{6}$ fue sobre niños sanos en edad escolar hace 8 años. Sin embargo, con el desarrollo del transporte y la atención médica, 
es posible que la epidemiología de $S$. aureus haya cambiado. El objetivo de este estudio consistió en describir la prevalencia de $S$. aureus, su resistencia a antibióticos y la presencia de los genes de LPV y $m e c A$ en niños en edad escolar que viven en zonas de gran altitud del sudoeste de China.

\section{POBLACIÓN Y MÉTODOS}

Este estudio se realizó entre octubre y noviembre de 2012 en Maerkang (altitud de 2500 a 4100 metros), una ciudad de la prefectura de Aba con una densidad de población de aproximadamente 9 personas por kilómetro cuadrado. En esta región rural, el acceso a los recursos médicos es relativamente escaso. Los antibióticos como la penicilina, la clindamicina y la eritromicina se utilizan en ocasiones, aunque la mayoría de los otros antibióticos no se emplean con frecuencia en esta población. En este estudio, se calculó que el tamaño de la muestra mínimo requerido para evaluar con precisión la incidencia de la portación de $S$. aureus en la región era de 233 , basado en la prevalencia prevista de la colonización nasal $(30 \%),{ }^{7}$ con un intervalo de confianza del $95 \%$.

Se reclutaron niños tibetanos sanos de escuelas primarias en la ciudad de Maerkang. Se excluyó a los niños que recibieron terapia con antibióticos o que requirieron hospitalización dentro de un período de 4 semanas antes del reclutamiento, así como también a los niños que sufrían infecciones cutáneas. Las muestras de hisopados se extrajeron girando dos veces un hisopo de algodón estéril, previamente humedecido con solución salina estéril, en el vestíbulo de cada orificio nasal anterior (narina) de los participantes, tras obtener el consentimiento informado de los padres de manera verbal.

Los hisopados nasales se transportaron al laboratorio empleando hisopos en tubos estériles con gel de agar Amies (Copan, Italia) y se inocularon en agar manitol salado (Oxoid Ltd, Basingstoke, Reino Unido) y en agar sangre Columbia (bioMérieux, Francia) durante 6 horas. A continuación, las inoculaciones se incubaron en $\mathrm{CO}_{2}$ al $5 \%$ durante $16-24$ horas a $35{ }^{\circ} \mathrm{C}$. La identificación de $S$. aureus se basó en la morfología de las colonias, en los resultados positivos de las pruebas de catalasa y de coagulasa en tubo, y en los resultados de un kit Slidex Staph Plus (bioMérieux, Francia). La sensibilidad antibiótica de las cepas aisladas se analizó mediante el método de difusión con discos, de acuerdo con las recomendaciones del Instituto de
Normas Clínicas y de Laboratorio (CLSI, por sus siglas en inglés), empleando los siguientes antibióticos: penicilina, eritromicina, clindamicina, gentamicina, cefoxitina y vancomicina. Todas las cepas aisladas de $S$. aureus se analizaron a fin de detectar el gen mecA mediante la prueba de la RCP para identificar un producto objetivo de $162 \mathrm{bp}$. Los partidores utilizados fueron mec $A$ P4 5'-TCCAGATTACAACTTCACCAGG-3' y mecA P 7 5'-CCACTTCATATCTTGTAACG-3' ${ }^{8}$ También se analizaron todas las cepas aisladas en busca de la presencia de genes de LPV empleando la prueba de la RCP con los partidores luk-pv1:5'ATCATTAGGTAAAATGTCTGGACATGATC CA-3' y luk-pv2:5'-GCATCAACTGTATTGGA TAGCAAAAGC-3'. ${ }^{9}$ Solamente las cepas que contenían el gen mec $A$ se clasificaron como SARM. ${ }^{10}$

Se realizó la prueba $\chi^{2}$ o la prueba exacta de Fisher para detectar la significación estadística $(5 \%)$ entre grupos mediante el software SPSS 13.0 para Windows (SPSS Inc., Chicago, IL, Estados Unidos de América). El comité médico del Segundo Hospital Universitario del Oeste de China, en Chengdú, otorgó la aprobación ética para este estudio el 28 de febrero de 2008.

\section{RESULTADOS}

En el estudio, participaron 314 niños tibetanos sanos, incluidos $182(58,0 \%)$ varones y $132(42,0 \%)$ mujeres. La edad promedio fue de 10,2 $\pm 1,2$ años (edades comprendidas entre los 6 y los 11 años), y el $44,6 \%$ de los participantes eran mayores de 10 años. Se aislaron cepas de $S$. aureus de 16 $(5,10 \%)$ niños; 10 eran varones y 6 , mujeres, sin significación estadística con respecto al sexo $(p=0,706)$. La resistencia de las cepas aisladas a la penicilina, eritromicina, clindamicina, rifampicina y cefoxitina fue del 100\%, 81,3\%,81,3\%, 0,0\% y $6,3 \%$, respectivamente. Se comprobó que la cepa resistente a la cefoxitina era SARM. Además, se detectó la expresión del gen mecA en 3 cepas aisladas, y 10 cepas aisladas dieron resultado positivo para el gen de LPV.

\section{DISCUSIÓN}

El S. aureus es uno de los principales microorganismos patógenos que, según se sabe, provoca una amplia variedad de enfermedades graves en los humanos, tanto intrahospitalarias como extrahospitalarias, y que abarcan desde infecciones cutáneas menores hasta infecciones graves, como la septicemia. ${ }^{4}$ La portación de $S$. aureus puede ser sumamente importante para 
el desarrollo de nuevas estrategias terapéuticas y medidas de prevención más eficaces. En este informe, se presentan datos sobre la prevalencia y la resistencia a antibióticos de las cepas aisladas de $S$. aureus, extraídas de la portación nasal en niños que viven en zonas de gran altitud del sudoeste de China. Según sabemos, este es el primer estudio que informa sobre la prevalencia de SARM en la población pediátrica de esta región.

Anteriormente, unos pocos estudios habían investigado la tasa de portación de S. aureus en regiones de poca altitud en la China continental. ${ }^{5}$ El equipo evaluó la prevalencia de la portación de $S$. aureus en niños sanos en edad escolar en Chengdú, China: fue del 18,35\%, y alrededor del $6 \%$ de las cepas aisladas de $S$. aureus se clasificaron como SARM entre 801 niños sanos. ${ }^{5}$ El presente estudio reveló que la tasa de portación nasal de $S$. aureus entre niños tibetanos sanos fue del $5,10 \%$. Esta tasa fue bastante baja en comparación con informes de estudios realizados en regiones de poca altitud. Como dato interesante, esta tasa es similar a una tasa notificada del $6,16 \%$ (22/357) de infecciones de las vías respiratorias bajas en niños tibetanos ${ }^{11} \mathrm{y}$ a una tasa notificada del 6,18\% (11/178) de hospitalizaciones en la unidad de cuidados intensivos pediátricos ${ }^{12}$ en una zona de gran altitud de China. Las tasas bajas de enfermedades pediátricas y de portación de S. aureus en esta región podrían estar relacionadas con la elevación del entorno, donde el aire es frío y enrarecido, y donde la densidad de población es baja. Si bien los niños provenientes de zonas con mejores condiciones económicas y mayor población (como Chengdú) por lo general cuentan con mayor acceso a la atención médica y reciben terapia con antibióticos de manera frecuente, las tasas bajas halladas en la población de este estudio podrían estar relacionadas con condiciones económicas y médicas deficientes. Sin embargo, esta relación necesita determinarse más detenidamente.

En este estudio, la tasa de portación nasal de S. aureus y SARM fue del $5,10 \%(16 / 314)$ y del $0,96 \%$ (3/314) en niños sanos, respectivamente. No obstante, en otro estudio ${ }^{6}$ realizado por este equipo hace 8 años, se demostró que la tasa de portación nasal de $S$. aureus fue del $2,4 \%$ en una población de 673 niños tibetanos sanos, en la que no se detectó ningún caso de SARM. Los resultados muestran que la tasa de $S$. aureus está aumentando en la zona y que debería prestarse atención al uso de antibióticos para S. aureus, especialmente en lo que respecta a SARM. Los resultados de este estudio indicaron que, ante casos de niños con infecciones graves por $S$. aureus, se debe optimizar el tratamiento con antibióticos sobre la base de una prueba de sensibilidad antibiótica. Asimismo, es necesario contar con vancomicina cuando se detecta SARM en cepas aisladas. Además, aunque la prevalencia de $S$. aureus sigue siendo baja, está aumentando en los niños sanos. Los datos sobre la prevalencia de $S$. aureus en zonas de gran altitud son escasos, ya que, por lo general, las investigaciones se centran en la China continental. Según se notificó en un estudio, ${ }^{13}$ el S. aureus provocó una tasa de mortalidad del 37,0\% en casos de septicemia neonatal, en una ciudad africana situada a gran altitud. Asimismo, es importante continuar investigando los factores ambientales que podrían contribuir a la incidencia de casos pediátricos de $S$. aureus en estas regiones.

Limitaciones del estudio

El presente estudio tuvo ciertas limitaciones. Debido a la baja densidad de población de la región, fue difícil reclutar a participantes que reunieran los requisitos. La ausencia de diferencias entre los sexos podría ser el resultado de la poca cantidad de pacientes. Además, el estudio epidemiológico de la portación nasal de S. aureus en esta región necesita examinarse más a fondo y seguirse de manera dinámica, ya que puede ser de gran importancia para esta región.

\section{CONCLUSIÓN}

Se detectó S. aureus en el 5,10\% (16/314) de la población del estudio; el 0,96\% (3/314) presentó SARM. Además, se detectó la expresión de los genes de LPV y mecA en 10 y 3 cepas aisladas, respectivamente.

\section{REFERENCIAS}

1. Song X, CogenJ, Singh N. Incidence of methicillin-resistant Staphylococcus aureus infection in a children's hospital in the Washington metropolitan area of the United States, 2003 - 2010. Emerg Microbes Infect 2013;2(10):e69.

2. Wertheim HF, Vos MC, Ott A, van Belkum A, et al. Risk and outcome of nosocomial Staphylococcus aureus bacteraemia in nasal carriers versus non-carriers. Lancet 2004;364(9435):703-5.

3. Dib J, Motok J, Zenoff VF, Ordoñez O, et al. Occurrence of resistance to antibiotics, UV-B, and arsenic in bacteria isolated from extremeenvironments in high-altitude (above 4400 m) Andean wetlands. Curr Microbiol 2008;56(5):510-7.

4. David MZ, Daum RS. Community-associated methicillinresistant Staphylococcus aureus: epidemiology and clinical consequences of an emerging epidemic. Clin Microbiol Rev 2010;23(3): 616-87.

5. Fan J, Shu M, Zhang G, Zhou W, et al. Biogeography and 
virulence of Staphylococcus aureus. PLoS One 2009;4(7):e6216.

6. Deng JJ, Xiao GG, Zhu Y, Zhou W, et al. Staphylococcus aureus Nasal Carriage and Its Antibiotic Resistance Profiles in TibetanSchoolChildren inSouthwest China. HKJPaediatr (new series) 2014;19:75-8.

7. Lamaro-Cardoso J, de Lencastre H, Kipnis A, Pimenta FC, et al. Molecular epidemiology and risk factors for nasal carriage of Staphylococcus aureus and methicillin-resistant S. aureus in infants attending day care centers in Brazil. J Clin Microbiol 2009;47(12):3991-7.

8. Oliveira DC, de Lencastre H. Multiplex PCR strategy for rapid identification of structural types and variants of the mec element in methicillin-resistant Staphylococcus aureus. Antimicrob Agents Chemother 2002;46(7):2155-61.

9. Lina G, Piémont $Y$, Godail-Gamont F, Bes $M$, et al. Involvement of Panton-Valentine leukocidin-producing
Staphylococcus aureus in primary skin infections and pneumonia. Clin Infect Dis 1999;29(5):1128-32.

10. Stegger M, Andersen PS, Keams A, Pichon B, et al. Rapid detection, differentiation and typing of methicillin-resistant Staphylococcus aureus harbouring either mecA or the new mecA homologue mecA(LGA251). Clin Microbiol Infect 2012;18(4):395-400.

11. XueH.Pathogenic analysis of Tibetan children in acute low respiratory tract infection in high-altitude areas. Zhongguo Xian Dai Yi Sheng 2015(26):76-8.

12. Xia YL, Ge M, Wang Z. Pathogenic analysis of ventilatorassociated pneumonia in the pediatric intensive care unit in high-altitude areas. Zhongguo Dang Dai Er Ke Za Zhi 2014;16(8):787-90.

13. Airede AI. Neonatal septicaemia in an African city of high altitude. J Trop Pediatr 1992;38(4):189-91.

\title{
Aplicación precoz de presión positiva continua en el tratamiento de la infección respiratoria aguda baja moderada-grave en pacientes menores de 2 años Early use of continuous positive airway pressure in the treatment of moderate to severe acute lower respiratory tract infections among patients younger than 2 years old
}

\author{
Dra. Laura Figueroa $a^{a}$ D Dr. Federico Laffaye
}

\section{RESUMEN}

Objetivo. Analizar las características de los pacientes menores de 2 años que padecieron una infección respiratoria aguda baja moderada-grave tratados precozmente con presión positiva continua en la vía aérea de burbuja y los factores asociados al éxito de la intervención.

Método. Estudio retrospectivo y descriptivo. Niños menores de 2 años internados en Cuidados Intermedios Pediátricos del Hospital Provincial Neuquén entre junio de 2009 y diciembre de 2010. Se aplicó presión positiva continua en la vía aérea de burbuja y se midieron frecuencias cardíacas y respiratorias, puntaje de Tal, saturación y fracción inspirada de oxígeno a las $0,2,6,24$ y $48 \mathrm{~h}$.

Resultados. Se incluyeron 120 pacientes. La mediana de edad fue 3 meses.

La intervención fue exitosa en el $72 \%$ de los casos. A las $2 \mathrm{~h}$, un descenso del $15 \%$ de la frecuencia respiratoria y de 2 puntos en el puntaje de Tal fueron predictores del éxito, con un OR 6,41 (IC 95\%:2,68-15,36) y 9,07 (IC 95\%:3,72-22,19), respectivamente.

a. Hospital Provincial Neuquén "Dr. E. Castro Rendón", Unidad de Cuidados Intermedios de Pediatría.

\section{Correspondencia:}

Dra. Laura Figueroa: lauritafigueroa@yahoo.com.ar

Financiamiento: Ninguno.

Conflicto de intereses: Ninguno que declarar.

Recibido: 9-5-2016

Aceptado: 26-12-2016
Conclusiones. La disminución de la frecuencia respiratoria, de la frecuencia cardíaca y del puntaje de Tal a las 2 horas de inicio fueron predictores de éxito.

Palabras clave: CPAP burbuja, bronquiolitis, neumonía, niños.

http:/ / dx.doi.org/10.5546/aap.2017.277

Texto completo en inglés:

http:/ / dx.doi.org/10.5546/aap.2017.eng.277

Cómo citar: Figueroa L, Laffaye F. Aplicación precoz de presión positiva continua en el tratamiento de la infección respiratoria aguda baja moderada-grave en pacientes menores de 2 años. Arch Argent Pediatr 2017;115(3):277-281.

\section{INTRODUCCIÓN}

Las infecciones respiratorias agudas bajas (IRAB) representan una importante causa de morbimortalidad en nuestro medio. ${ }^{1}$

Entre las principales entidades clínicas incluidas en las IRAB, se encuentran la bronquiolitis y la neumonía, que son más importantes por su impacto en la mortalidad infantil, ${ }^{2} \mathrm{y}$, sobre estas, se basa el presente trabajo.

Colaboradores:

Mario González, Germán Kaltenbach, Viviana Arias y Eugenia Cerda. 